This is the final peer-reviewed accepted manuscript of:

M. Fabrizio; B. Lazzari; R. Nibbi - Existence and stability for a visco-plastic material with a fractional constitutive equation -

Mathematical Methods in the Applied Sciences, vol. 40 (2017), pp. 6306-6315.

The final published version is available online at: https://doi.org/10.1002/mma.4458

Rights / License:

The terms and conditions for the reuse of this version of the manuscript are specified in the publishing policy. For all terms of use and more information see the publisher's website.

This item was downloaded from IRIS Università di Bologna (https://cris.unibo.it/)

When citing, please refer to the published version. 


\title{
Existence and stability for a visco-plastic material with a fractional constitutive equation
}

\author{
M. Fabrizio*, B. Lazzari, R. Nibbi
}

The well posedness of the evolutive problem for visco-plastic materials represented by two different fractional constitutive equations is proved. We show that, for these materials, we can observe permanent deformations. So that, as it is usual in plasticity, when the stress goes to zero, then the strain assumes a constant non zero behavior. Moreover, we prove the compatibility of our models with the classical laws of thermodynamics. For the second model, described through a fractional derivative with an exponential kernel, we obtain the exponential decay of the solutions by means of the semigroup theory. Copyright (c) 2009 John Wiley \& Sons, Ltd.

Keywords: Visco-plasticity; fractional derivative; asymptotic behavior; stability.

\section{Introduction}

In this paper, we study the existence, uniqueness and asymptotic behavior of a visco-plastic material described by a constitutive equation with memory, which makes use of the fractional Caputo derivative ${ }_{a}^{C} D_{t}^{\alpha}[1,2]$. In this framework, the material is described by the constitutive equation

$$
\boldsymbol{\sigma}(x, t)=\mathbf{A}(x){ }_{a}^{C} D_{t}^{\alpha} \boldsymbol{\varepsilon}(x, t)=\frac{\mathbf{A}(x)}{\Gamma(1-\alpha)} \int_{a}^{t} \frac{\frac{\partial}{\partial \tau} \boldsymbol{\varepsilon}(x, \tau)}{(t-\tau)^{\alpha}} d \tau,
$$

where $\boldsymbol{\sigma}$ denotes the stress, $\boldsymbol{\varepsilon}=\frac{1}{2}\left[\nabla \mathbf{u}+(\nabla \mathbf{u})^{T}\right]=\operatorname{sym}\{\nabla \mathbf{u}\}$ the infinitesimal strain tensor, while $\Gamma$ is the gamma function, A a suitable symmetric and positive definite tensor and $a \leq 0$. Finally, $\alpha$ is a scalar parameter, which defines the degree of the Caputo fractional derivative.

The results presented in this paper hold for $\alpha \in(0,1)$; however, we observe that the constitutive equation (1.1) describes plastic solid materials if $\alpha \in\left(0, \frac{1}{2}\right)$. The plastic behavior of this model is evident when we work with strain processes defined by

$$
\boldsymbol{\varepsilon}(x, t)=\boldsymbol{\varepsilon}_{0}(x) \neq 0, \quad t>t_{0}>0 .
$$

Indeed, for these processes, from (1.1), we have

$$
\lim _{t \rightarrow \infty} \boldsymbol{\sigma}(x, t)=\lim _{t \rightarrow \infty} \frac{\mathbf{A}(x)}{\Gamma(1-\alpha)} \int_{a}^{t} \frac{\frac{\partial}{\partial \tau} \boldsymbol{\varepsilon}^{\prime}(x, \tau)}{(t-\tau)^{\alpha}} d \tau=0,
$$

describing a visco-plastic behavior of the material, because the stress goes to zero, even if the deformation remains constant and different from zero.

This analysis suggests that the study of an equation with memory of the type (1.1) needs a certainly different approach from that followed for viscoelastic materials with fading memory [13, 14], as fatigue phenomena [3, 11].

So that, it is suitable to observe that, for visco-plastic solid materials with a constitutive equation (1.1), with $0<\alpha<<\frac{1}{2}$, the natural unknown is not the displacement $\mathbf{u}$, but its fractional derivative, because the permanent deformations, due to the visco-plastic nature of the material, involve different asymptotic behaviors for $\mathbf{u}$ towards the zero solution. 
This new position requires a different formulation of the initial value problem as presented in Section 2

In the second part of the work, we propose a different constitutive equation which makes use of a new definition of fractional derivative presenting an exponential memory kernel (see [5]). In this model the plastic effects are faster to stabilize, compared with constitutive equation (1.1), because the memory effects are less evident.

Finally, for this model, following [9], we prove the exponential decay of the solutions.

\section{Free energy and Dissipation Law}

In this paper, we prefer to use a different formulation of the Caputo fractional derivative.

Let $f$ be a function belonging to a suitable subset of the bounded continuous functions on $(a, \infty)$ with $f(a)=0$ and consider its Caputo fractional derivative:

$$
{ }_{a}^{C} D_{t}^{(\alpha)} f(t)=\frac{1}{\Gamma(1-\alpha)} \int_{a}^{t} \frac{\frac{d}{d \tau} f(\tau)}{(t-\tau)^{\alpha}} d \tau
$$

If $f$ is Hölder continuous with exponent $\beta>\alpha$, an integration by parts of (2.1) leads to

$$
{ }_{a}^{c} D_{t}^{(\alpha)} f(t)=\frac{1}{\Gamma(1-\alpha)}\left[\frac{f(t)}{(t-a)^{\alpha}}+\alpha \int_{a}^{t} \frac{f(t)-f(\tau)}{(t-\tau)^{1+\alpha}} d \tau\right], t>a .
$$

By observing that

$$
\frac{1}{(t-a)^{\alpha}}=\int_{-\infty}^{a} \frac{\alpha}{(t-\tau)^{1+\alpha}} d \tau
$$

and extending the function $f$ to $\mathbb{R}$ as follows

$$
{ }_{a} f(\tau)=\left\{\begin{array}{cl}
f(\tau), & \tau \geq a \\
0, & -\infty<\tau<a
\end{array},\right.
$$

the operator (2.1) can be rewritten in the following way

$$
{ }_{a}^{C} D_{t}^{(\alpha)} f(t)=\frac{\alpha}{\Gamma(1-\alpha)} \int_{-\infty}^{t} \frac{f(t)-{ }_{a} f(\tau)}{(t-\tau)^{1+\alpha}} d \tau \quad t>0 .
$$

In the sequel, we restrict $\boldsymbol{\varepsilon}$ to a set of sufficiently smooth functions and consider the following formulation for the constitutive equation (1.1)

$$
\begin{aligned}
\boldsymbol{\sigma}(x, t) & =\frac{\alpha \mathbf{A}(x)}{\Gamma(1-\alpha)} \int_{-\infty}^{t} \frac{\boldsymbol{\varepsilon}(x, t)-{ }_{a} \boldsymbol{\varepsilon}(x, \tau)}{(t-\tau)^{1+\alpha}} d \tau \\
& =\frac{\alpha \mathbf{A}(x)}{\Gamma(1-\alpha)} \int_{0}^{\infty} \frac{\boldsymbol{\varepsilon}(x, t)-{ }_{a} \boldsymbol{\varepsilon}^{t}(x, s)}{s^{1+\alpha}} d s, \quad t>0 .
\end{aligned}
$$

In this model, the state in a point $x$ of the body at the time $t$ is given by

$$
{ }_{a} \boldsymbol{S}^{t}(x, \cdot)=\boldsymbol{\varepsilon}(x, t)-{ }_{a} \boldsymbol{\varepsilon}^{t}(x, \cdot),
$$

where ${ }_{a} \boldsymbol{\varepsilon}^{t}(\cdot, s)={ }_{a} \boldsymbol{\varepsilon}(\cdot, t-s)$ is the extended history of the strain tensor.

Since it is well known the strict connection between the thermodynamic restrictions and stability conditions for the evolutive problem, in this section we study the expression of the free energy and the related Dissipation Law.

Dissipation Law There exists a state function $\psi$, called free energy, such that

$$
\rho_{0}(x) \frac{\partial}{\partial t} \psi\left({ }_{a} s^{t}(x, \cdot)\right) \leq \boldsymbol{\sigma}(x, t) \cdot \frac{\partial}{\partial t} \boldsymbol{\varepsilon}(x, t) .
$$

for any mechanical process.

Taking into account the stress constitutive equation (2.4), (2.5) becomes

$$
\rho_{0}(x) \frac{\partial}{\partial t} \psi\left({ }_{a} \boldsymbol{s}^{t}(x, \cdot)\right) \leq \frac{\alpha \mathbf{A}(x)}{\Gamma(1-\alpha)} \int_{0}^{\infty} \frac{{ }_{a} \boldsymbol{s}^{t}(x, s)}{s^{1+\alpha}} d s \cdot \frac{\partial}{\partial t} \boldsymbol{\varepsilon}(x, t)
$$

and, as proved in [8], we can define the following free energy functional

$$
\rho_{0}(x) \psi\left({ }_{a} \boldsymbol{s}^{t}(x, \cdot)\right)=\frac{\alpha}{2 \Gamma(1-\alpha)} \int_{0}^{\infty} \frac{\mathbf{A}(x){ }_{a} \boldsymbol{s}^{t}(x, s) \cdot{ }_{a} \boldsymbol{s}^{t}(x, s)}{s^{1+\alpha}} d s
$$


which leads to the dissipation

$$
D(x, t)=\frac{\alpha(1+\alpha)}{2 \Gamma(1-\alpha)} \int_{0}^{\infty} \frac{\mathbf{A}(x){ }_{a} \boldsymbol{s}^{t}(x, s) \cdot{ }_{a} \boldsymbol{s}^{t}(x, s)}{s^{2+\alpha}} d s \geq 0
$$

In fact, we can prove that

$$
\rho_{0}(x) \frac{\partial}{\partial t} \psi\left({ }_{a} \boldsymbol{s}^{t}(x, \cdot)\right)=\frac{\alpha \mathbf{A}(x)}{\Gamma(1-\alpha)} \int_{0}^{\infty} \frac{{ }_{a} \boldsymbol{s}^{t}(x, s)}{s^{1+\alpha}} d s \cdot \frac{\partial}{\partial t} \boldsymbol{\varepsilon}(x, t)-\frac{\alpha(1+\alpha)}{2 \Gamma(1-\alpha)} \int_{0}^{\infty} \frac{\mathbf{A}(x){ }_{a} \boldsymbol{s}^{t}(x, s) \cdot{ }_{a} \boldsymbol{s}^{t}(x, s)}{s^{2+\alpha}} d s
$$

and we get (2.6) thanks to (2.8).

Finally, from the symmetry of the tensor $\mathbf{A}(x)$, the free energy assumes the new form

$$
\rho_{0}(x) \psi\left({ }_{a} \boldsymbol{s}^{t}(x, \cdot)\right)=\frac{\alpha}{2 \Gamma(1-\alpha)} \int_{0}^{\infty} \frac{\mathbf{A}(x)\left[\nabla \mathbf{u}(x, t)-\nabla_{a} \mathbf{u}^{t}(x, s)\right] \cdot\left[\nabla \mathbf{u}(x, t)-\nabla_{a} \mathbf{u}^{t}(x, s)\right]}{s^{1+\alpha}} d s .
$$

\section{Differential problem via Caputo fractional model}

Let $\Omega$ be a bounded domain in $\mathbb{R}^{3}$ with a smooth boundary. We consider the classical momentum balance differential equation

$$
\rho_{0}(x) \frac{\partial^{2}}{\partial t^{2}} \mathbf{u}(x, t)=\nabla \cdot \boldsymbol{\sigma}(x, t)+\rho_{0}(x) \mathbf{b}(x, t)
$$

where $\rho_{0}$ denotes the density, $\mathbf{u}$ the displacement vector and $\mathbf{b}$ the external supply.

Now, we connect equation (3.1) with the constitutive equation (2.4), the homogeneous boundary condition

$$
\left.\mathbf{u}(x, t)\right|_{\partial \Omega}=0
$$

and the initial conditions

$$
\mathbf{u}(x, 0)=\mathbf{u}_{0}(x), \frac{\partial}{\partial t} \mathbf{u}(x, 0)=\mathbf{v}_{0}(x), \nabla \mathbf{u}^{t=0}(x, s)=\nabla \mathbf{u}_{0}(x, s), a<s<0,
$$

where $\nabla \mathbf{u}^{t=0}$ denotes the past history of $\nabla \mathbf{u}$ in $t=0$.

Without loss in generality, we can limit ourselves to study a problem with homogeneous initial conditions and initially null external sources. To this aim let us introduce an auxiliary smooth function $\mathbf{z}$ with the following properties:

$$
\begin{aligned}
& \mathbf{z}_{\mid \partial \Omega}(x, t)=\mathbf{0}, \quad \mathbf{z}(x, 0)=\mathbf{u}_{0}(x), \quad \frac{\partial}{\partial t} \mathbf{z}(x, 0)=\mathbf{v}_{0}(x), \\
& \frac{\partial^{2}}{\partial t^{2}} \mathbf{z}(x, 0)=\mathbf{b}(x, 0)+\frac{\alpha}{\rho_{0}(x) \Gamma(1-\alpha)} \nabla \cdot \int_{a}^{0} \frac{\mathbf{A}(x) \nabla \mathbf{u}_{0}(x, s)}{s^{(1+\alpha)}} d s, \\
& \mathbf{z}(x, t)=0 \quad \text { for } t \geq t_{0} .
\end{aligned}
$$

The function $\widetilde{\mathbf{u}}=\mathbf{u}-\mathbf{z}$ it is solution of the problem

$$
\begin{aligned}
& \rho_{0}(x) \frac{\partial^{2}}{\partial t^{2}} \widetilde{\mathbf{u}}(x, t)=\frac{\alpha}{\Gamma(1-\alpha)} \nabla \cdot \int_{0}^{\infty} \frac{\mathbf{A}(x) \nabla\left[\widetilde{\mathbf{u}}(x, t)-{ }_{0} \widetilde{\mathbf{u}}^{t}(x, s)\right]}{s^{1+\alpha}} d s+\rho_{0}(x) \mathbf{f}(x, t) \\
& \widetilde{\mathbf{u}}(x, 0)=\mathbf{0}, \quad \frac{\partial}{\partial t} \widetilde{\mathbf{u}}(x, 0)=\mathbf{0},\left.\quad \widetilde{\mathbf{u}}(x, t)\right|_{\partial \Omega}=\mathbf{0} .
\end{aligned}
$$

where

$$
\begin{aligned}
\rho_{0}(x) \mathbf{f}(x, t)= & \rho_{0}(x) \mathbf{b}(x, t)+\frac{\alpha}{\Gamma(1-\alpha)} \nabla \cdot \int_{a}^{0} \frac{\mathbf{A}(x) \nabla \mathbf{u}_{0}(x, s)}{(t-s)^{(1+\alpha)}} d s \\
& -\rho_{0}(x) \frac{\partial^{2}}{\partial t^{2}} \mathbf{z}(x, t) \frac{\alpha}{\Gamma(1-\alpha)} \nabla \cdot \int_{0}^{t} \frac{\mathbf{A}(x) \nabla[\mathbf{z}(x, t)-\mathbf{z}(x, \tau)]}{(t-\tau)^{1+\alpha}} d \tau
\end{aligned}
$$

and $\mathbf{f}(x, 0)=\mathbf{0}$

Moreover, thanks to the properties of $\mathbf{z}, \widetilde{\mathbf{u}}(x, t)=\mathbf{u}(x, t)$ for $t \geq t_{0}$. Therefore, in what follows, we restrict our attention to problem (3.4).

As observed in the Introduction, for this problem permanent deformations are evident. Indeed, in presence of a source $\mathbf{f}$ with $\lim _{t \rightarrow \infty} \mathbf{f}(x, t)=0$ it is possible to find a function $\mathbf{u}$, solution of problem (3.4) such that

$$
\operatorname{sym}\left\{\nabla \widetilde{\mathbf{u}}_{\infty}(x)\right\}=\lim _{t \rightarrow \infty} \operatorname{sym}\{\nabla \widetilde{\mathbf{u}}(x, t)\}=\lim _{t \rightarrow \infty} \operatorname{sym}\{\nabla \mathbf{u}(x, t)\}=\operatorname{sym}\left\{\nabla \mathbf{u}_{\infty}(x)\right\} \neq 0 .
$$


Incidentally, we observe that $\operatorname{sym}\left\{\nabla \widetilde{\mathbf{u}}_{\infty}\right\}$ coincides with $\operatorname{sym}\left\{\nabla \mathbf{u}_{\infty}\right\}$ of the original problem (3.1)-(3.2) and denotes the viscoplastic deformation depending on the initial conditions and the supply process $\mathbf{b}$.

For this reason, it appears reasonable for materials described by equation (1.1), to state the problem using as unknown the function

$$
\mathbf{w}(x, t)=\frac{\alpha}{\Gamma(1-\alpha)} \int_{0}^{\infty} \frac{\widetilde{\mathbf{u}}(x, t)-{ }_{0} \widetilde{\mathbf{u}}^{t}(x, s)}{s^{1+\alpha}} d s:=\widetilde{\mathbf{u}}^{(\alpha)}(x, t) .
$$

So, by observing that the null initial conditions assure that

$$
\frac{\partial}{\partial t} \widetilde{\mathbf{u}}=\mathbf{w}^{(1-\alpha)}
$$

system (3.4) can be rewritten as follows

$$
\begin{aligned}
& \rho_{0}(x) \frac{\partial}{\partial t}\left[\mathbf{w}^{(1-\alpha)}(x, t)\right]=\nabla \cdot[\mathbf{A}(x) \nabla \mathbf{w}(x, t)]+\rho_{0}(x) \mathbf{f}(x, t) \\
& \mathbf{w}(x, 0)=\mathbf{0}, \quad \mathbf{w}^{(1-\alpha)}(x, 0)=\mathbf{0} \\
& \left.\mathbf{w}(x, t)\right|_{\partial \Omega}=0
\end{aligned}
$$

From now on, for simplicity, we suppose $\rho_{0}(x)=1$ and denote with $H_{0}^{\alpha}\left(\mathbb{R}^{+}\right)$the space

$$
H_{0}^{\alpha}\left(\mathbb{R}^{+}\right)=\left\{f \in L^{2}\left(\mathbb{R}^{+}\right) ;{ }_{0}^{c} D_{t}^{\alpha} f \in L^{2}\left(\mathbb{R}^{+}\right), f(0)=0\right\} .
$$

Definition 3.1 A function $\mathbf{w} \in H_{0}^{1-\alpha}\left(\mathbb{R}^{+} ; L^{2}(\Omega)\right) \cap L^{2}\left(\mathbb{R}^{+} ; H_{0}^{1}(\Omega)\right)$ is a weak solution to problem (3.5) if it satisfies the integral equality

$$
\begin{gathered}
\left.\int_{0}^{\infty} \int_{\Omega}\left[\mathbf{w}^{(1-\alpha)}(x, t) \cdot \frac{\partial}{\partial t} \boldsymbol{\phi}(x, t)\right)-\mathbf{A}(x) \nabla \mathbf{w}(x, t) \cdot \nabla \boldsymbol{\phi}(x, t)\right] d x d t \\
=-\int_{0}^{\infty} \int_{\Omega} \mathbf{f}(x, t) \cdot \boldsymbol{\phi}(x, t) d x d t
\end{gathered}
$$

for all $\phi \in H_{0}^{1}\left(\mathbb{R}^{+} ; L^{2}(\Omega)\right) \cap L^{2}\left(\mathbb{R}^{+} ; H_{0}^{1}(\Omega)\right)$.

Let us consider the causal Fourier transform of equation (3.5). For $\omega>0$, we obtain

$$
\begin{aligned}
& (i \omega)^{2-\alpha} \hat{\mathbf{w}}(x, \omega)=\nabla \cdot[\mathbf{A}(x) \nabla \hat{\mathbf{w}}(x, \omega)]+\hat{\mathbf{f}}(x, \omega) \\
& \hat{\mathbf{w}}(x, \omega)_{\mid \partial \Omega}=0
\end{aligned}
$$

We multiply both sides of $(3.7)$ by $\left[(i \omega)^{1-\alpha} \hat{\mathbf{w}}(x, \omega)\right]^{*}$, where the symbol ${ }^{*}$ denotes the complex conjugate, and integrate over the domain $\Omega$.

Since

$$
\left[(i \omega)^{1-\alpha}\right]^{*}=\left(\cos \frac{(1-\alpha) \pi}{2}-i \sin \frac{(1-\alpha) \pi}{2}\right) \omega^{1-\alpha},
$$

after using the boundary condition, we get

$$
\begin{aligned}
& i \omega \int_{\Omega}\left|(i \omega)^{(1-\alpha)} \hat{\mathbf{w}}(x, \omega)\right|^{2} d x+\int_{\Omega}\left[\cos \frac{(1-\alpha) \pi}{2}-i \sin \frac{(1-\alpha) \pi}{2}\right] \omega^{1-\alpha} \mathbf{A}(x) \nabla \hat{\mathbf{w}}(x, \omega) \cdot \nabla \hat{\mathbf{w}}^{*}(x, \omega) d x \\
& =\int_{\Omega}\left[(i \omega)^{1-\alpha}\right]^{*} \hat{\mathbf{f}}(x, \omega) \cdot \hat{\mathbf{w}}^{*}(x, \omega) d x .
\end{aligned}
$$

The real and imaginary components of (3.8) provide

$$
\begin{gathered}
\omega^{1-\alpha} \cos \frac{(1-\alpha) \pi}{2}\|\hat{\mathbf{w}}(\omega)\|_{A}^{2}=\Re\left[\int_{\Omega}\left[(i \omega)^{1-\alpha}\right]^{*} \hat{\mathbf{f}}(x, \omega) \cdot \hat{\mathbf{w}}^{*}(x, \omega) d x\right] \\
\omega\left\|(i \omega)^{1-\alpha} \hat{\mathbf{w}}(\omega)\right\|^{2}-\sin \frac{(1-\alpha) \pi}{2} \omega^{1-\alpha}\|\hat{\mathbf{w}}(\omega)\|_{A}^{2}=\Im\left[\int_{\Omega}\left[(i \omega)^{1-\alpha}\right]^{*} \hat{\mathbf{f}}(x, \omega) \cdot \hat{\mathbf{w}}^{*}(x, \omega) d x\right],
\end{gathered}
$$

where

$$
\|\hat{\mathbf{w}}(\omega)\|_{A}^{2}=\int_{\Omega} \mathbf{A} \nabla \hat{\mathbf{w}}(x, \omega) \cdot \nabla \hat{\mathbf{w}}^{*}(x, \omega) d x
$$

is equivalent to the usual norm in $H_{0}^{1}(\Omega)$. 
Then, from (3.9) we have

$$
\cos \frac{(1-\alpha) \pi}{2}\|\hat{\mathbf{w}}(\omega)\|_{A}^{2} \leq\left[\int_{\Omega}|\hat{\mathbf{f}}(x, \omega)|\left|\hat{\mathbf{w}}^{*}(x, \omega)\right| d x\right]
$$

and the Poincaré inequality leads to

$$
\cos \frac{(1-\alpha) \pi}{2}\|\hat{\mathbf{w}}(\omega)\|_{A} \leq C_{1}\|\hat{\mathbf{f}}(\omega)\|, \quad \cos \frac{(1-\alpha) \pi}{2}\|\hat{\mathbf{w}}(\omega)\| \leq C_{2}\|\hat{\mathbf{f}}(\omega)\|
$$

with $C_{1}$ and $C_{2}$ positive constants depending on the domain $\Omega$.

By using the Plancherel theorem we can reach the following result:

Theorem 3.2 If $\mathbf{f} \in H_{0}^{1-\alpha}\left(\mathbb{R}^{+} ; L^{2}(\Omega)\right)$ then problem (3.5) admits one and only one weak solution.

Proof. If $\mathbf{f} \in H_{0}^{1-\alpha}\left(\mathbb{R}^{+} ; L^{2}(\Omega)\right)$ then $\|\hat{\mathbf{f}}(\omega)\|$ and $\left\|\omega^{(1-\alpha)} \hat{\mathbf{f}}(\omega)\right\|$ belong to $L^{2}(\mathbb{R})$. Since $\cos \frac{(1-\alpha) \pi}{2} \neq 0$, inequalities (3.11) assure that $\|\hat{\mathbf{w}}(\omega)\|_{A} \in L^{2}\left(\mathbb{R}^{+}\right)$and $\left\|\omega^{(1-\alpha)} \hat{\mathbf{w}}(\omega)\right\| \in L^{2}\left(\mathbb{R}^{+}\right)$.

From the Plancherel theorem it follows that $\mathbf{w} \in H_{0}^{1-\alpha}\left(\mathbb{R}^{+} ; L^{2}(\Omega)\right) \cap L^{2}\left(\mathbb{R}^{+} ; H_{0}^{1}(\Omega)\right)$.

\section{A new fractional model for a visco-plastic material}

In this section, we consider a new type of fractional derivative operator proposed in [4]

$$
{ }_{a} \mathcal{D}_{t}^{(\alpha)} f(t)=\frac{1}{1-\alpha} \int_{a}^{t} e^{-\frac{\alpha(t-\tau)}{1-\alpha}} \frac{d}{d \tau} f(\tau) d \tau
$$

where $a \in[-\infty, 0], \alpha \in[0,1]$, the function $f$ belongs to a subset of $C(a, \infty)$ with $f(a)=0$.

It is possible to prove that, when $\alpha=1$, this fractional derivative coincides with the classical first derivative, whereas it is the identity operator if $\alpha=0$.

We shall now proceed as in Section 2. Observing that

$$
\frac{1}{1-\alpha}=\int_{-\infty}^{t} e^{-\frac{\alpha(t-\tau)}{1-\alpha}} d \tau
$$

after an integration by parts the operator (4.1) can be rewritten as follows

$$
{ }_{a} \mathcal{D}_{t}^{(\alpha)} f(t)=\frac{\alpha}{(1-\alpha)^{2}} \int_{-\infty}^{t} e^{-\frac{\alpha(t-\tau)}{1-\alpha}}\left[f(t)-{ }_{a} f(\tau)\right] d \tau, t>a
$$

We now consider visco-plastic materials where the stress $\sigma$ is related to this new fractional derivative of the infinitesimal strain through the constitutive equation

$$
\begin{aligned}
\boldsymbol{\sigma}(x, t) & =\frac{1}{1-\alpha} \mathbf{A}(x) \int_{-\infty}^{t} e^{-\frac{\alpha(t-\tau)}{1-\alpha}} \frac{\partial}{\partial \tau} \boldsymbol{\varepsilon}(x, \tau) d \tau \\
& =\frac{\alpha}{(1-\alpha)^{2}} \mathbf{A}(x) \int_{0}^{\infty} e^{-\frac{\alpha(t-\tau)}{1-\alpha}}\left[\boldsymbol{\varepsilon}(x, t)-{ }_{a} \boldsymbol{\varepsilon}(x, t-s)\right] d s
\end{aligned}
$$

with A symmetric and positive definite tensor.

Also for this model, the state in a point $x$ of the body at the time $t$ is given by ${ }_{a} \boldsymbol{s}^{t}(x, \cdot)=\boldsymbol{\varepsilon}(x, t)-{ }_{a} \boldsymbol{\varepsilon}^{t}(x, \cdot)$.

To test the compatibility of the constitutive equation (4.3) with the Dissipation Principle, we introduce the functional $\Psi$, depending on ${ }_{a} \boldsymbol{s}^{t}$, as follows

$$
\rho_{0}(x) \Psi\left({ }_{a} \boldsymbol{s}^{t}(x, \cdot)\right)=\frac{\alpha}{2(1-\alpha)^{2}} \int_{0}^{\infty} e^{-\frac{\alpha(t-\tau)}{1-\alpha}} \mathbf{A}(x){ }_{a} s^{t}(x, s) \cdot{ }_{a} s^{t}(x, s) d s
$$

and prove that $\psi$ is a free energy functional which satisfies the inequality (2.5). Observing that

$$
\frac{\partial}{\partial t}{ }_{a}{ }^{t}(x, s)=\frac{\partial}{\partial t} \boldsymbol{\varepsilon}(x, t)+\frac{\partial}{\partial s}{ }_{a} \boldsymbol{\varepsilon}^{t}(x, s),
$$


the time derivative of $(4.4)$ is

$$
\begin{aligned}
\rho_{0}(x) \frac{\partial}{\partial t} \Psi\left({ }_{a} \boldsymbol{s}^{t}(x, \cdot)\right)= & \frac{\alpha}{(1-\alpha)^{2}} \int_{0}^{\infty} e^{-\frac{\alpha(t-\tau)}{1-\alpha}} \mathbf{A}(\mathbf{x})_{a} \boldsymbol{s}^{t}(x, s) d s \cdot \frac{\partial}{\partial t}(\boldsymbol{\varepsilon}(x, t) \\
& +\frac{\alpha}{(1-\alpha)^{2}} \int_{0}^{\infty} e^{-\frac{\alpha(t-\tau)}{1-\alpha}} \mathbf{A}(\mathbf{x}){ }_{a} \boldsymbol{s}^{t}(x, s) \cdot \frac{\partial}{\partial s}{ }_{a} \boldsymbol{s}^{t}(x, s) d s \\
= & \boldsymbol{\sigma}(x, t) \cdot \frac{\partial}{\partial t} \boldsymbol{\varepsilon}(x, t)+\frac{\alpha}{2(1-\alpha)^{2}} \int_{0}^{\infty} e^{-\frac{\alpha(t-\tau)}{1-\alpha}} \frac{\partial}{\partial s}\left[\mathbf{A}(\mathbf{x})_{a} \boldsymbol{s}^{t}(x, s) \cdot{ }_{a} \boldsymbol{s}^{t}(x, s)\right] d s \\
= & \boldsymbol{\sigma}(x, t) \cdot \frac{\partial}{\partial t} \boldsymbol{\varepsilon}(x, t)-\frac{\alpha^{2}}{2(1-\alpha)^{3}} \int_{0}^{\infty} e^{-\frac{\alpha(t-\tau)}{1-\alpha}} \mathbf{A}(\mathbf{x})_{a} \boldsymbol{s}^{t}(x, s) \cdot{ }_{a} \boldsymbol{s}^{t}(x, s) d s \\
= & \boldsymbol{\sigma}(x, t) \cdot \frac{\partial}{\partial t} \boldsymbol{\varepsilon}(x, t)-\frac{\alpha}{1-\alpha} \rho_{0}(x) \Psi\left({ }_{a} \boldsymbol{s}^{t}(x, \cdot)\right)
\end{aligned}
$$

so that the dissipation $D$ is given by

$$
D(t)=\frac{\alpha}{1-\alpha} \rho_{0}(x) \Psi\left({ }_{a} \varsigma^{t}(x, \cdot)\right)
$$

\section{Exponential energy decay}

In this section we study the differential problem (3.1)-(3.2), introduced in Section 3, associated with the constitutive equation (4.3) for $\boldsymbol{\sigma}$.

According to the approach followed in Section 3, we introduce the unknown, related to the fractional derivative, defined in (4.1) and consider the function

$$
\boldsymbol{w}(x, t)=\mathbf{u}^{[\alpha]}(x, t):={ }_{0} \mathcal{D}_{t}^{(\alpha)} \mathbf{u}(x, t)=\frac{\alpha}{(1-\alpha)^{2}} \int_{-\infty}^{t} e^{-\frac{\alpha(t-\tau)}{1-\alpha}}[\mathbf{u}(x, t)-\mathbf{u}(x, \tau)] d \tau .
$$

Using the same mathematical technique as in Section 3, we can restrict our attention to the following problem with homogeneous initial conditions

$$
\begin{aligned}
& \frac{\partial}{\partial t}\left[\boldsymbol{w}^{[1-\alpha]}(x, t)\right]=\nabla \cdot[\mathbf{A}(x) \nabla \boldsymbol{w}(x, t)]+\mathbf{f}(x, t) \\
& \boldsymbol{w}(x, 0)=\mathbf{0}, \quad \boldsymbol{w}^{[1-\alpha]}(x, 0)=\mathbf{0} \\
& \left.\boldsymbol{w}(x, t)\right|_{\partial \Omega}=0
\end{aligned}
$$

with

$$
\mathbf{f}(x, t)=\mathbf{b}(x, t)+\nabla \cdot\left[\frac{\alpha}{(1-\alpha)^{2}} \int_{a}^{0} e^{-\frac{\alpha}{1-\alpha}(t-\tau)} \mathbf{A}(x) \nabla[\mathbf{u}(x, t)-\mathbf{u}(x, \tau)] d \tau\right]
$$

and $\rho_{0}=1$.

Denoting with $\mathcal{H}_{0}^{\alpha}\left(\mathbb{R}^{+}\right)$the space

$$
\mathcal{H}_{0}^{\alpha}\left(\mathbb{R}^{+}\right)=\left\{f \in L^{2}\left(\mathbb{R}^{+}\right) ;{ }_{0} \mathcal{D}_{t}^{(\alpha)} f \in L^{2}\left(\mathbb{R}^{+}\right), f(0)=0\right\}
$$

we give the following definition

Definition 5.1 A function $w \in \mathcal{H}_{0}^{1-\alpha}\left(\mathbb{R}^{+} ; L^{2}(\Omega)\right) \cap L^{2}\left(\mathbb{R}^{+} ; H_{0}^{1}(\Omega)\right)$ is a weak solution to problem (5.1) if it satisfies the integral equality

$$
\int_{0}^{\infty} \int_{\Omega}\left[\boldsymbol{w}^{[1-\alpha]}(x, t) \cdot \frac{\partial}{\partial t} \boldsymbol{\phi}(x, t)-\mathbf{A}(x) \nabla \boldsymbol{w}(x, t) \cdot \nabla \boldsymbol{\phi}(x, t)\right] d x d t=-\int_{0}^{\infty} \int_{\Omega} \mathbf{f}(x, t) \cdot \boldsymbol{\phi}(x, t) d x d t
$$

for all $\phi \in H_{0}^{1}\left(\mathbb{R}^{+} ; L^{2}(\Omega)\right) \cap L^{2}\left(\mathbb{R}^{+} ; H_{0}^{1}(\Omega)\right)$.

To obtain the existence and uniqueness result, we proceed as in the previous Section 3 and study the the causal Fourier transform of system (5.1) for $\omega>0$.

It is easy to prove that the causal Fourier transform of the fractional derivative of a function $f \in \mathcal{H}_{0}^{\beta}\left(\mathbb{R}^{+}\right)$is given by

$$
\widehat{f[\beta]}(\omega)=\frac{i \omega}{\beta-i \omega(1-\beta)} \hat{f}(\omega)=\frac{\omega[(1-\beta) \omega+i \beta]}{\beta^{2}+(1-\beta)^{2} \omega^{2}} \hat{f}(\omega)
$$


Applying the causal Fourier to equation $(5.1)_{1}$, we obtain

$$
\begin{aligned}
& i \omega \frac{i \omega}{(1-\alpha)-i \alpha \omega} \hat{\boldsymbol{w}}(x, \omega)=\nabla \cdot[\mathbf{A}(x) \nabla \hat{\boldsymbol{w}}(x, \omega)]+\hat{\mathbf{f}}(x, \omega) \\
& \hat{\boldsymbol{w}}(x, \omega)_{\mid \partial \Omega}=0
\end{aligned}
$$

If we multiply both sides of $(5.3)_{1}$ by $\left(\widehat{w^{[1-\alpha]}}\right)^{*}$ and integrate on the domain $\Omega$, we get

$$
i \omega\left\|\frac{i \omega}{(1-\alpha)-i \alpha \omega} \hat{\boldsymbol{w}}(\omega)\right\|^{2}-\frac{i \omega}{(1-\alpha)+i \alpha \omega}\|\hat{\boldsymbol{w}}(\omega)\|_{A}^{2}=-\frac{i \omega}{(1-\alpha)+i \alpha \omega} \int_{\Omega} \hat{\mathbf{f}}(x, \omega) \cdot \hat{w}^{*}(x, \omega) d x .
$$

The real and imaginary components of (5.4) provide

$$
\begin{gathered}
\alpha \omega^{2}\|\hat{\boldsymbol{w}}(x, \omega)\|_{A}^{2}=\Re\left[-\omega[\alpha \omega+i(1-\alpha)] \int_{\Omega} \hat{\mathbf{f}}(x, \omega) \cdot \hat{\boldsymbol{w}}^{*}(x, \omega) d x\right], \\
\omega^{3}\|\hat{\boldsymbol{w}}(x, \omega)\|^{2}-(1-\alpha) \omega\|\hat{\boldsymbol{w}}(x, \omega)\|_{A}^{2}=\Im\left[-\omega[\alpha \omega+i(1-\alpha)] \int_{\Omega} \hat{\mathbf{f}}(x, \omega) \cdot \hat{\boldsymbol{w}}^{*}(x, \omega) d x\right] .
\end{gathered}
$$

Then, from (5.5) and (5.6) we have

$$
\begin{array}{r}
\|\hat{\boldsymbol{w}}(x, \omega)\|_{A}^{2} \leq \frac{|\alpha \omega+i(1-\alpha)|}{\alpha \omega}\|\hat{\mathbf{f}}(\omega)\|\|\hat{\boldsymbol{w}}(\omega)\| \\
\|\hat{\boldsymbol{w}}(x, \omega)\|_{A}^{2} \leq \frac{|\alpha \omega+i(1-\alpha)|}{1-\alpha}\|\hat{\mathbf{f}}(\omega)\|\|\hat{\boldsymbol{w}}\|+\omega^{2}\|\hat{\boldsymbol{w}}(x, \omega)\|^{2}
\end{array}
$$

Since

by Poincare inequality, we obtain

$$
\lim _{\omega \rightarrow 0} \frac{|\alpha \omega+i(1-\alpha)|}{1-\alpha}=1, \quad \lim _{\omega \rightarrow \infty} \frac{|\alpha \omega+i(1-\alpha)|}{\alpha \omega}=1,
$$

$$
\|\hat{\boldsymbol{w}}(\omega)\|_{A} \leq K\|\hat{\mathbf{f}}(\omega)\|, \quad \forall \omega
$$

with $K>0$. The inequality (5.8) assures that $w \in L^{2}\left(\mathbb{R}^{+} ; H_{0}^{1}(\Omega)\right)$.

If we require $\mathbf{f} \in \mathcal{H}_{0}^{1-\alpha}\left(\mathbb{R}^{+} ; L^{2}(\Omega)\right)$, then $\boldsymbol{w} \in \mathcal{H}_{0}^{1-\alpha}\left(\mathbb{R}^{+} ; L^{2}(\Omega)\right)$ or, equivalently, $w^{[1-\alpha]} \in L^{2}\left(\mathbb{R}^{+} ; L^{2}(\Omega)\right)$.

The previous results can be summarized in the following theorem:

Theorem 5.2 If $\mathbf{f} \in \mathcal{H}_{0}^{1-\alpha}\left(\mathbb{R}^{+} ; L^{2}(\Omega)\right)$ then problem (5.1) admits one and only one weak solution according to the Definition 5.1.

The result on the exponential decay of the energy is established by using the theory of contraction semigroups in Hilbert spaces. We only provide a very brief outline, because our problem is a particular case of a more general class of material with memory studied, for example, in [9].

First we introduce the Hilbert space $\mathcal{K}\left(\mathbb{R}^{+}, \Omega\right)$ of the pairs

$$
\chi(x, t)=\left(w^{[1-\alpha]}(x, t),{ }_{a} s^{t}(x, \cdot)\right)
$$

with the inner product

$$
\begin{aligned}
<\chi_{1}(t), \chi_{2}(t)>=\int_{\Omega} \boldsymbol{w}_{1}^{[1-\alpha]}(x, t) \cdot \boldsymbol{w}_{2}^{[1-\alpha]}(x, t) d x \\
\quad+\frac{\alpha}{(1-\alpha)^{2}} \int_{\Omega} \int_{0}^{\infty} e^{-\frac{\alpha s}{1-\alpha}} \mathbf{A}(x) \boldsymbol{\varsigma}_{a_{1}}^{t}(x, s) \cdot \boldsymbol{\varsigma}_{a_{2}}^{t}(x, s) d s d x<\infty,
\end{aligned}
$$

so that the associated norm is the energy norm, i.e.

$$
\mathcal{E}(t)=\frac{1}{2}<\chi(t), \chi(t)>=\frac{1}{2}\left\|w^{[1-\alpha]}\right\|^{2}+\int_{\Omega} \Psi\left({ }_{a} \boldsymbol{s}^{t}(x, \cdot)\right) d x
$$

Then we rewrite problem (3.1)-(3.2), associated to the constitutive equation (4.3) for $\boldsymbol{\sigma}$, as an abstract Cauchy problem as follows

$$
\begin{aligned}
\frac{\partial}{\partial t} \boldsymbol{w}^{[1-\alpha]}(x, t) & =\frac{\alpha}{(1-\alpha)^{2}} \nabla \cdot \mathbf{A}(x) \int_{0}^{\infty} \exp \left[-\frac{\alpha s}{1-\alpha}\right]{ }_{a} \boldsymbol{s}^{t}(x, s) d s \\
\frac{\partial}{\partial t}{ }_{a} \boldsymbol{s}^{t}(x, s) & =\nabla \boldsymbol{w}^{[1-\alpha]}(x, t)+\frac{\partial}{\partial s}{ }_{a} \boldsymbol{s}^{t}(x, s) \\
\boldsymbol{w}^{[1-\alpha]}(x, 0) & =\mathbf{v}_{0}(x), \quad{ }_{a} \boldsymbol{s}^{t=0}(x, s)={ }_{a} \boldsymbol{s}^{0}(x, s), s \geq 0
\end{aligned}
$$


or, in a compact form,

$$
\frac{\partial}{\partial t} \chi(x, t)=\mathcal{A}(x) \chi(x, t), \quad \chi(x, 0)=\chi_{0}(x)
$$

The domain of the operator $\mathcal{A}$ is the dense subset of $\mathcal{K}\left(\mathbb{R}^{+}, \Omega\right)$ defined as follows

$$
\mathcal{D}(\mathcal{A})=\left\{\chi \in \mathcal{K}\left(\mathbb{R}^{+}, \Omega\right) ; \mathcal{A} \chi \in \mathcal{K}\left(\mathbb{R}^{+}, \Omega\right)\right\} .
$$

Since

$$
\frac{1}{2}<\mathcal{A} \chi(t), \chi(t)>=\frac{\partial}{\partial t} \mathcal{E}(t)=-\frac{\alpha}{1-\alpha} \int_{\Omega} \Psi\left({ }_{a} s^{t}(x, \cdot)\right) d x,
$$

employing standard techniques, we can affirm that $\mathcal{A}$ generates a strongly continuous semigroup of linear contractions $\mathcal{S}(t)$ on $\mathcal{K}\left(\mathbb{R}^{+}, \Omega\right)$ with respect to the energy norm so that the solution of (5.11) can be written in the form $\chi(t)=\mathcal{S}(t) \chi_{0}$ and $\mathcal{E}(t)=\frac{1}{2}<\mathcal{S}(t) \chi_{0}, \mathcal{S}(t) \chi_{0}>$.

Finally, the equality (5.12) and the previous Theorem 5.2 assure that

$$
\int_{0}^{\infty} \mathcal{E}(t) d t<\infty
$$

The exponential decay of the energy follows from classical theorems of semigroups theory (see, for example, [12]).

\section{Acknowledgments}

The authors has been partially supported by GNFM - INdAM and by the University of Bologna.

The authors declare that they have no conflict of interest.

\section{References}

1. M. Caputo, Linear models of dissipation whose $Q$ is almost frequency independent, part II, Geophys. J. R. Astron. Soc., 13. (1967), 529-539; http://dx.doi.org/10.1111/j.1365-246X.1967.tb02303.x

2. M. Caputo, A model for the fatigue in elastic materials with frequency independent Q, J. Acoust. Soc. Am., 66 No 1 , (1979), 176-179; http://dx.doi.org/10.1121/1.383058

3. M. Caputo and M. Fabrizio, Damage and fatigue described by a fractional derivative model, J. Comput. Phys., 293, (2015), 400-408; http://dx.doi.org/10.1016/j.jcp.2014.11.012

4. M. Caputo, M. Fabrizio, A new Definition of Fractional Derivative without Singular Kernel, Progr. Fract. Differ. Appl., 1, No. 2, (2015), 73-85; http://dx.doi.org/10.12785/pfda/010201

5. M. Caputo, M. Fabrizio, Applications of new time and spatial fractional derivatives with exponential kernels, Progr. Fract. Differ. Appl., 2, No. 1, (2016), 1-11; http://dx.doi.org/10.18576/pfda/020101

6. M. Di Paola, M. Zingales, Exact mechanical models of fractional hereditary materials, J. Rheol., 56, (2012), 983-1004; http://dx.doi.org/10.1122/1.4717492

7. M. Fabrizio, Fractional rheological models for thermomechanical systems. Dissipation and free energies. Fract. Calc. Appl. Anal., 17, No. 1, (2014), 206-223; http://dx.doi.org/10.2478/s13540-014-0163-7

8. M Fabrizio, B Lazzari, On asymptotic stability for linear viscoelastic fluids, Differential Integral Equations, 6, No. 3 , (1993) 491-505;

http://projecteuclid.org/euclid.die/1370378426

9. M. Fabrizio, B. Lazzari, On the existence and the asymptotic stability of solutions for linearly viscoelastic solids, Arch. Rational Mech. Anal. 116, (1991), 139-152; http://dx.doi.org/10.1007/BF00375589

10. M. Fabrizio, B. Lazzari, R. Nibbi, Thermodynamics of non-local materials: extra fluxes and internal powers. Contin. Mech. Thermodyn. 23, No. 6, (2011), 509-525; http://dx.doi.org/10.1007/s00161-011-0193-x

11. M. Fremond and B. Nedjar, Damage, gradient of damage and principle of virtual power, Int. J. Solids Structures, 33, (1996), 1083-1103; http://dx.doi.org/10.1016/0020-7683(95)00074-7

12. A. Pazy, Semigroups of linear operators and applications to partial differential equations, Applied Mathematical Sciences, 44. Springer-Verlag, New York, (1983);http://dx.doi.org/10.1007/978-1-4612-5561-1

13. Y. Povstenko, Fractional Thermoelasticity, vol. 219 of Solid Mechanics and Its Applications, Springer (2015); http://dx.doi.org/10.1007/978-3-319-15335-3

14. H. H. Sherief, A.M.A. El-Sayed and A.M.A. El-Latief, Fractional order theory of thermoelasticity, Intern. J. Solids and Structures, 47, (2010), 269-275; http://dx.doi.org/10.1016/j.ijsolstr.2009.09.034 